Review

\title{
Nickel and Epigenetic Gene Silencing
}

\section{Hong Sun ${ }^{1}$, Magdy Shamy ${ }^{2}$ and Max Costa ${ }^{1, *}$}

1 Department of Environmental Medicine, NYU School of Medicine, Tuxedo, NY 10987, USA;

\author{
E-Mail: Hong.Sun@nyumc.org
}

2 Department of Environmental Sciences, Faculty of Meteorology, Environment and Arid Land Agriculture, King Abdulaziz University, Jeddah 21589, Saudi Arabia;

E-Mail: Madgyshamy@hotmail.com

* Author to whom correspondence should be addressed; E-Mail: Max.Costa@nyumc.org; Tel.: +1-845-731-3515; Fax: +1-845-351-2118.

Received: 21 May 2013; in revised form: 11 October 2013 / Accepted: 17 October 2013 / Published: 25 October 2013

\begin{abstract}
Insoluble nickel compounds are well-established human carcinogens. Occupational exposure to these compounds leads to increased incidence of lung and nasal cancer in nickel refinery workers. Apart from its weak mutagenic activity and hypoxia mimicking effect there is mounting experimental evidence indicating that epigenetic alteration plays an important role in nickel-induced carcinogenesis. Multiple epigenetic mechanisms have been identified to mediate nickel-induced gene silencing. Nickel ion is able to induce heterochromatinization by binding to DNA-histone complexes and initiating chromatin condensation. The enzymes required for establishing or removing epigenetic marks can be targeted by nickel, leading to altered DNA methylation and histone modification landscapes. The current review will focus on the epigenetic changes that contribute to nickel-induced gene silencing.
\end{abstract}

Keywords: epigenetics; gene silencing; heterochromatin; DNA methylation; histone modification; miRNA

\section{Introduction}

Nickel (Ni) is one of the most abundant elements in our planet. Ni compounds are extensively used in numerous industrial processes, including the production of coins, jewelry, stainless steel, medical 
devices, Ni-Cd batteries, and $\mathrm{Ni}$ refinery, etc. Occupational exposure to $\mathrm{Ni}$ compounds through inhalation has become a big health concern for workers involved in the different stages of $\mathrm{Ni}$ processing [1,2]. Epidemiological studies have reported an increased incidence of lung and nasal cancer among nickel refinery workers [3-6]. It is worth noting that almost all nickel refinery workers smoked cigarettes, suggesting a possible synergistic effect of insoluble nickel compounds and cigarette smoking in cancer induction. In addition, insoluble chronic exposure to $\mathrm{Ni}$ compounds induced tumor formation in various animal models, further supporting the carcinogenic activity of nickel compounds [7-9]. Moreover, both water-soluble and insoluble compounds can transform cultured mammalian cells in vitro [10-13]. In 1990, the International Agency for Research on Cancer (IARC) classified all Ni (II) compounds as known human carcinogens (group 1) and metallic nickel as possible human carcinogens (group 2b) [13-15].

Multiple mechanisms have been proposed to mediate Ni-induced carcinogenesis, including its genotoxic and mutagenic activity, hypoxia-mimicking effects, dysregulation of cell signaling, and alterations of the epigenetic landscape [16-18]. DNA strand breaks and chromosomal aberration have been observed in Ni-exposed cells [19]. Compared to active euchromatic regions, transcriptionally inactive heterochromatic regions are more susceptible targets of Ni-induced damage. An early study from our laboratory demonstrated that $\mathrm{Ni}$ compounds selectively damage the heterochromatic long arm of the X chromosome in Chinese hamster ovary ( $\mathrm{CHO}$ ) cells [20]. The insoluble nickel compounds green nickel oxide and crystalline nickel monosulfide induce amplification of the ect-2 proto-oncogene in $\mathrm{C} 3 \mathrm{H} / 10 \mathrm{~T} 1 / 2 \mathrm{Cl} 8$ mouse embryo fibroblasts, which is found in green $\mathrm{NiO}$ and crystalline NiS-transformed 10T1/2 cells. Hence, the genotoxic activity of insoluble nickel compounds, in terms of gene amplification, is likely part of the molecular mechanisms by which nickel compounds induce morphological and neoplastic cell transformation [21]. In addition to its genotoxicity, Ni compounds are able to stabilize hypoxia inducible factors (HIFs) by inhibiting the activity of the HIF prolyl- and asparaginyl-hydroxylases [22,23]. Ni induced HIFs stabilization activates HIF-dependent transcription and initiates a series of hypoxia-specific responses under normoxia condition [24]. As a common phenomenon in many solid tumors, hypoxia arises in rapidly growing tumors due to the limitation of oxygen diffusion, and further facilitates tumor progression and metastasis. Thus, the hypoxia-mimicking effect of $\mathrm{Ni}$ compounds has been considered as an important mechanism underlying Ni-induced carcinogenesis [16].

Recent advances in cancer research have shown that epigenetic alterations play an important role in tumor formation and progression $[25,26]$. In the past two decades, research conducted in our laboratory and others have established the role of $\mathrm{Ni}$ in modulating the epigenetic landscape [16,27]. Ni is able to target the epigenetic machinery and induce alterations in chromatin structure, DNA methylation and histone modifications.

\section{Epigenetics and Gene Silencing}

Epigenetics refers to the reversible but inheritable changes in gene expression that occur without alterations in DNA sequence [28]. In eukaryotes, genomic DNA is tightly packed with histone proteins to form a highly dynamic structure called chromatin. Depending on the degree of DNA condensation, chromatin can be divided into two different forms: heterochromatin (closed form, highly condensed 
region with little or no transcriptional activity) and euchromatin (open form, less condensed region with more active transcription). These "open" and "closed" chromatin domains are important for the accessibility of genetic information and have significant impacts in chromatin related biological functions, such as DNA replication, DNA recombination, DNA repair, transcription activation and repression, transcription initiation, elongation and termination, etc. [28].

The basic structural unit of chromatin is the nucleosome, which is composed of $\sim 147$-bp double strand DNA wrapped around an octamer core with two sets of histone proteins H2A, H2B, H3 and H4. The N-terminal tail of each histone protein protrudes from the nucleosome and serves as a platform for various chemical modifications. The histone modifications cooperate with other epigenetic factors including DNA methylation and small non-coding RNAs, not only influencing local chromatin structure but also playing an important role in DNA accessibility and gene expression.

\subsection{Histone Modification}

The N-terminal tails of four histone proteins can be modified by various post-translational modifications at more than 60 different residues. While lysine residues can be modified by acetylation, methylation, sumoylation, ubiquitination and biotinylation, arginine can be modified by methylation, citrullination and ADP-ribosylation. Other modifications include phosphorylation of serine and threonine, ADP-ribosylation of glutamic acid, and cis-trans isomerization of proline [29]. Depending on the position and the protein bound, the modifications may have different functions on chromatin remodeling and gene expression [29].

Among these modifications, lysine acetylation and methylation have been extensively studied. Acetylation of lysine residues removes the positive charge from their side chains and decreases the affinity between histone tails and DNA, which subsequently leads to increased DNA accessibility and transcription activation in the promoter region of genes. Acetyl groups can be transferred to lysine residues by histone acetyltransferases (HATs) and removed by histone deacetylases (HDAC). These enzymes are normally associated with different large multi-protein complexes to either activate (HATs) or repress (HDACs) gene transcription [29,30].

In addition to acetylation, lysine residues can be mono-, di- or tri-methylated. Unlike acetylation, methylation of lysine does not change the positive charge on the side chain [29]. Therefore, depending on the position of the lysine residue and the number of methyl groups, lysine methylation can be associated with either active or repressive transcription. For example, di- and tri- methylation of histone H3 lysine 9 (H3K9) are repressive marks. While tri-methylated H3K9 (H3K9me3) is associated with heterochromatic regions, di-methylated $\mathrm{H} 3 \mathrm{~K} 9$ (H3K9me2) is found mostly in euchromatic regions and linked to the promoter of silenced genes. Other well-studied methylation marks include methylated H3K4 (active marks), tri-methylated H3K27 (a repressive mark) and methylation of H3K36 (a mark associated with transcription elongation) [29,31].

Similar to histone acetylation, lysine methylation is tightly controlled by a number of methyltransferases and demethylases. Most lysine methyltransferases (KMTs) contains a Set domain, and use S-adenosyl methionine (SAM) as the methyl donor. Two major types of lysine demethylases (KDMs) were identified. Lysine-specific demethylase 1(LSD1) is a flavin-dependent monoamine oxidase, which removes mono- or di-methyl groups from $\mathrm{H} 3 \mathrm{~K} 4$ and $\mathrm{H} 3 \mathrm{~K} 9$ using FAD as a co-factor. 
Another type of lysine demethylase belongs to a big family of jmjc-domain containing dioxygenases that have more than 27 family members. Jmjc-doamin containing demethylases require oxygen, 2-oxoglutarate and iron as co-factors, and are able to remove mono-, di-, and tri-methyl groups from many different lysine residues [31].

\subsection{DNA Methylation}

DNA methylation is a covalent modification in which a methyl group is added to 5-position of cytosine. In mammalian cells, the majority of 5-methylcytosine $(5 \mathrm{mC})$ is located in the context of CpG dinucleotides. About 60-90\% CpGs are methylated in mammalian cells, and the methylation pattern correlates with long term transcriptional silencing, such as genomic imprinting, X-chromosome inactivation, suppression of repetitive elements, as well as maintaining lineage specific gene silencing. It is worth noting that, while DNA methylation in the promoter is typically linked to transcription repression, gene body methylation is more likely correlated to transcription activation [32].

Due to its essential role in maintaining genomic stability and modulating gene expression, $5 \mathrm{mC}$ levels are dynamically regulated by DNA methylation and demethylation [33]. First, cytosine methylation is catalyzed by DNA methyltransferases (DNMTs), a family of enzymes that is able to transfer the methyl group from SAM to cytosine. While DNMT3A and DNMT3B establish 5mC patterns in germ cells and developing embryos via de novo DNA methylation, DNMT1 and its partner (nuclear protein 95) specifically bind to hemimethylated DNA during DNA replication and copy 5mC marks from the parental strand to the newly synthesized strand [34]. Second, methyl groups can be gradually removed from cytosines across generations by lack of DNA methyltransferases or their activities during DNA replication. This process is called passive DNA demethylation and it depends on cell division and contributes to the global demethylation in the maternal pronuclei of the zygote. Lastly, recent advances have identified an active DNA demethylation process that involves the ten-eleven translocation (TET) methylcytosine dioxygenase, activation-induced cytidine deaminase (AID) and thymine DNA glycosylase (TDG) [35]. During active demethylation, $5 \mathrm{mC}$ is first oxidized by TET proteins using iron, oxygen and 2-oxoglutarate as co-factors. This reaction converts $5 \mathrm{mC}$ to 5-hydroxymethylcytosine (5hmC), 5-formylcytosine (5fC), and 5-carboxylcytosine $(5 \mathrm{caC})$, which can be subsequently removed by AID or TDG and base excision repair system [35]. Active demethylation mechanism has been implicated in reprogramming of the DNA methylation profile in primordial germ cells and the paternal pronuclei of the zygote.

How does DNA methylation suppress gene transcription? DNA methylation in gene promoter regions may interfere with the binding of transcription factors that are required for gene activation. Long-term gene silencing may be mediated by a group of proteins that contain a methyl-binding domain (MBD). MBD proteins recruit co-repressors such as HDACs, and other chromatin modifing enzymes to form compact and repressive chromatin. There are five MBD proteins, MeCP2, MBD1, MBD2, MBD3 and MBD4, and each MBD protein recruits different sets of protein partners and represses different target genes. Recent genome-wide studies confirmed the correlation between DNA methylation and chromatin accessibility [32]. 


\section{Nickel and Gene Silencing}

Evidence of $\mathrm{Ni}$-induced gene silencing first emerged toward the end of the 1980s. Conway and Costa $[19,20]$ reported that the incidence of nickel-induced transformation is about $2-3$-fold higher in male Chinese Hamster Embryonic (CHE) cells than those in female CHE cells, while there was no gender difference in 3-methylcholanthrene (MCA) induced CHE transformation. Karyotype analysis revealed that the majority of nickel-transformed cells exhibited either deletion or translocation of the long arm of the $\mathrm{X}$ chromosome $(\mathrm{Xq})$, suggesting a possible role of the $\mathrm{X}$ chromosome in Ni-induced cell transformation of CHE cells. Interestingly, re-introducing the normal Chinese Hamster X chromosome into nickel-transformed cells resulted in cell senescence [36], indicating inactivation of one or several unknown genes located on the $\mathrm{X}$ chromosome as a prerequisite for Ni-induced transformation of CHE cells.

The ability of nickel to silence gene expression was soon confirmed using a transgene system in which the endogenous hypoxanthine-guanine phosphoribosyltransferase (HPRT) gene in Chinese Hamster V79 cells was mutated by UV light and replaced by a small bacteria xanthine-guanine phosphoribosyl transferase (gpt) gene [37]. The cells that carry the gpt transgene can be selected by the resistance to either 6-thioguanine (6TG) for gpt inactive cells or HAT (hypoxanthine, aminopterin, and thymidine) medium for gpt active cells. One of the transgene cell lines, G12, has the gpt gene inserted near the telomere region of chromosome 1 which is close to a long stretch of heterochromatin. G12 cells exhibit a high level of 6TG resistance after exposure to water-insoluble Ni compounds (nickel sulfide, nickel subsulfide, and nickel oxides) or long term exposure to a water-insoluble Ni compound (nickel chloride), indicating $\mathrm{Ni}$ exposure is able to silence the gpt transgene [38,39]. Interestingly, $\mathrm{Ni}$-induced gpt gene silencing can also be observed in an additional transgenic cell line, G10, however, gpt silencing in G10 cells was much less efficient compared to that in G12 cells. The difference between G12 and G10 is that gpt gene was inserted near a euchromatic region of chromosome 6 in G10 cells [39], indicating that the location of the transgene is critical for Ni-induced gene silencing.

In addition to transgene, several endogenous genes were silenced in $\mathrm{C} 3 \mathrm{H} / 10 \mathrm{~T} 1 / 2$ mouse embryo cells transformed by green $\mathrm{NiO}$ and crystalline $\mathrm{NiS}$, including the vitamin $\mathrm{D}$ receptor interacting protein 80 (DRIP80) gene, the insulin-like growth factor 1 (IGFR1) gene, the small nuclear activating protein C3 (SNAP C3) gene, the b-centaurin-2 gene and FAD synthetase gene [40-42].

\subsection{Nickel and Heterochromatinization}

Two approaches were designed to further address whether the location of the gpt transgene contributes to its sensitivity to $\mathrm{Ni}$-induced silencing. First, heterochromatin fraction was isolated from G10, G12 and a 6-TG resistant clone derived from Ni-treated G12 cells and analyzed for the amount of gpt transgene. The amount of gpt transgene was highly enriched in the heterochromatin fraction from either G12 cells or G12-derived 6-TG resistant clone, but not in G10 cells. In Ni-treated G12 clones, the portion of the gpt gene associated with the heterochromatin fraction was significantly increased compared to those in parental G12 cells [43]. This result indicated that the heterochromatin location of the gpt gene in G12 cells not only contributes to its silencing induced by nickel, but also sensitizes the cells to Ni-induced silencing. The second approach used DNase I to probe the higher order of 
chromatin structure surrounding the gpt transgene in freshly isolated nuclei. A sensitive DNase I PCR method revealed an immediate DNA condensation in G12 nuclei following $1 \mathrm{~h}$ nickel treatment in vitro, which was not seen in G10 nuclei. Also, the sequence surrounding the gpt transgene exhibited a time-dependent increase in condensation following Ni treatment [43]. More interestingly, Ni-treated clones derived from G12 cells are more resistant to DNase I digestion [43]. These effects on G12 cells were inhibited by excess magnesium ion. These results indicate that nickel treatment is able to induce chromatin condensation near heterochromatic region, a process called heterochromatinization, which may inactivate nearby genes, such as the gpt transgene or tumor suppressor genes (Figure 1A).

Figure 1. Schematic models for nickel mediated gene silencing. (A) Nickel increased local heterochromatinization and DNA methylation led to inactivation of nearby genes (Adapted from Ellen 2009 [44], @2009 American Chemical Society). (B) Transcription active genes exhibited an open active chromatin with less DNA methylation and more active histone mark histone $\mathrm{H} 3$ acetylation (H3ac). Nickel treatment converted it to an inactive chromatin by inducing repressive mark $\mathrm{H} 3$ lysine 9 dimethylation (H3K9me2) and DNA methylation $(5 \mathrm{mC})$ as well as inhibiting $\mathrm{H} 3$ acetylation.

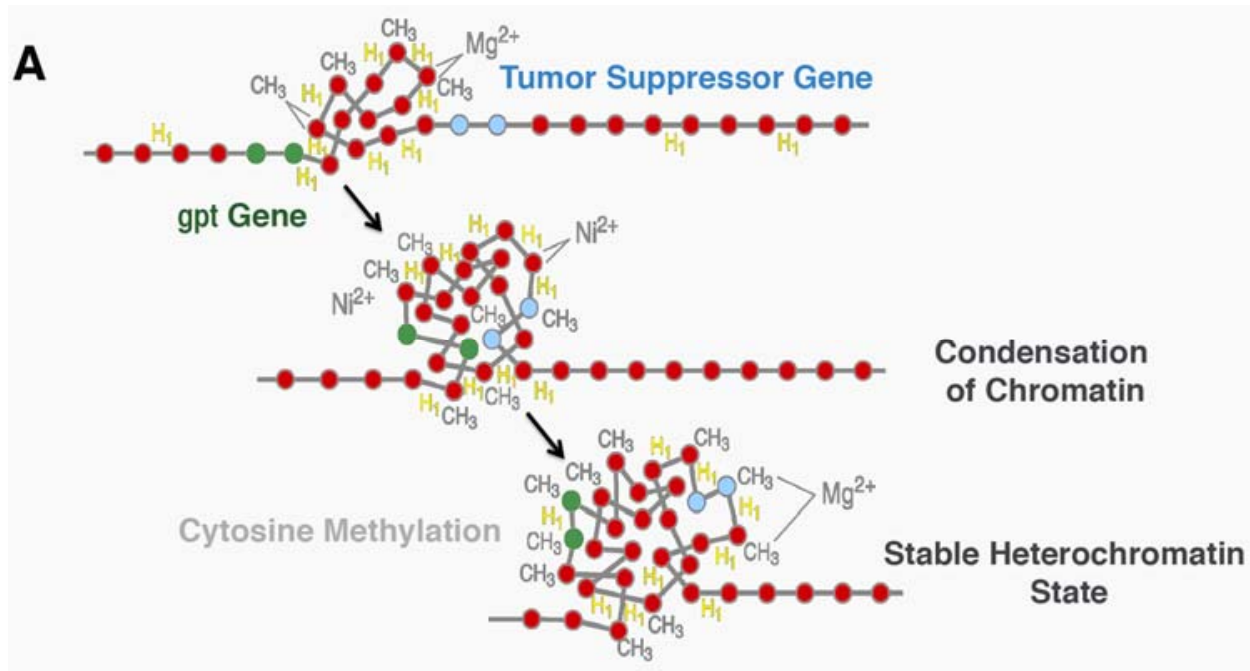

B

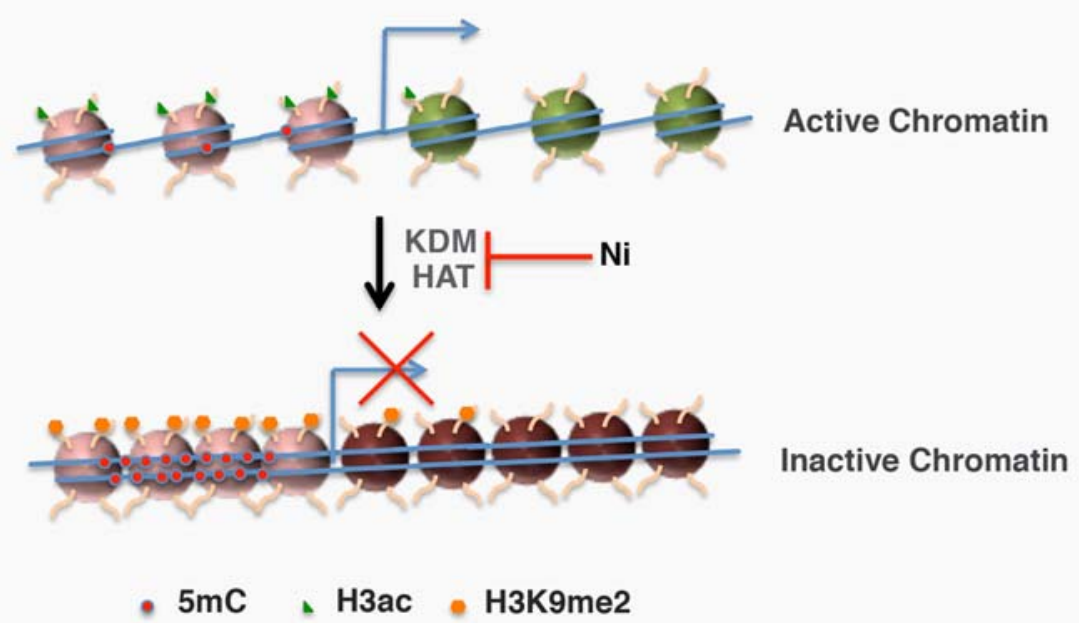

Involvement of heterochromatinization in Ni-induced gene silencing was soon confirmed by reconstitution of nucleosomal array using atomic force microscopy [44]. In the same concentration, 
nickel-exposed oligonucleosomes exhibited a much greater condensation compared to those exposed to magnesium. Similar results were obtained by circular dichroism spectropolarimetry, indicating that nickel-treated oligonucleosomes form a more compact higher-order chromatin structure than magnesium-treated oligonucleosomes [44]. Moreover, nickel treatment enhanced the resistance of the gpt transgene to DNase I digestion in G12 cells (gpt transgene inserted near heterochromatin) but not in G10 cells (gpt transgene inserted in euchromatin). The resistance was not seen in G12 cells treated with cobalt and was much weaker in magnesium-treated cells [43]. This data, together with our earlier studies, indicates that Ni-mediated heterochromatinization plays an important role in Ni-induced gene silencing.

\subsection{Nickel and DNA Methylation}

Other than heterochromatinization, DNA methylation is the first epigenetic factor found to contribute to Ni-induced gene silencing.

In G12 cells, the gpt transgene silenced by nickel sulfide can be reactivated by treating cells with 5-aza-cytidine, a strong inhibitor of DNA methyltransferase. DNA hypermethylation was confirmed in the gpt transgene using methylation sensitive restriction enzymes [43]. Moreover, chromatin immunoprecipitation showed that methyl-binding protein $\mathrm{MeCP} 2$ was enriched at the gpt transgene in nickel silenced clones. This enrichment was not seen in parental G12 cells [45]. These results indicated that nickel exposure was able to induce local DNA methylation that subsequently silenced the gpt transgene.

In addition to the gpt transgene, DNA methylation has been associated with $\mathrm{Ni}$-induced silencing of endogenous genes in cultured cells. Nickel exposure of human bronchial epithelial (BEAS-2B) cells induced epithelial-mesenchymal transition (EMT). E-cadherin, which encodes a cell surface adhesion glycoprotein, was silenced in Ni-treated cells by DNA hypermethylation in its promoter region [46]. Similarly, $\mathrm{O}^{6}$-methylguanine DNA methyltransferase (MGMT), an enzyme that repairs $\mathrm{O}^{6}$-methylguanine, was silenced by promoter hypermethylation in NiS-transformed human bronchial epithelial (16HBE) cells [47]. Recent studies on the Syrian hamster (SHD) cell lines immortalized by soluble nickel demonstrated that hypermathylation of $\mathrm{CpG}$ cluster immediately upstream of p16 exon 1 resulted in p16 gene silencing and consequent cell senescence bypass [48].

$\mathrm{Ni}$-induced promoter hypermethylation was also observed in vivo. Nickel sulfide was implanted into p53 heterozygous (p53+/-) mice to induce tumor formation. Malignant fibrous histiocytomas developed in both wild type and $\mathrm{p} 53+/-$ mice, and all the tumors exhibited promoter hypermethylation of the tumor suppressor gene p16 [49]. Moreover, intramuscular injection of nickel subsulfide to Wistar rats induced muscle tumors that displayed DNA hypermethylation in the promoter regions of RAR $\beta 2$, RASSF1A and p16 genes [50]. Thus, DNA hypermethylation and subsequent silencing of tumor suppressor genes may serve as an epigenetic mechanism mediating nickel's carcinogenicity (Figure 1A).

\subsection{Nickel and Histone Modification}

In addition to DNA methylation, local histone modification may also contribute to Ni-induced gene silencing. Histone acetylation is normally associated with gene activation. Exposure to $\mathrm{Ni}$ and $\mathrm{Ni}$ compounds reduced the global levels of acetylated lysine in all four histones in yeast, rat and human cells [51-53]. Nickel is able to inhibit histone $\mathrm{H} 4$ acetylation in vitro, suggesting that HAT activity 
may be one target of nickel [51,54]. In vitro activity assay confirmed a dose-dependent inhibition of HAT activity by nickel chloride [54]. Most interestingly, addition of the antioxidants in the reaction significantly reversed nickel induced inhibition of HAT activity, while co-treatment with hydrogen peroxide led to more inhibition [54]. It has been shown that nickel exposure increased reactive oxygen species (ROS) production and initiated the cell response to oxidative stress $[55,56]$. These results suggested a role of oxidative stress in nickel induced epigenetic changes. In addition, TSA, a potent HDAC inhibitor, is able to reduce the $\mathrm{Ni}$-induced gene silencing in both yeast and mammalian cells [57], indicating that HDAC may be involved in Ni-induced gene silencing. Moreover, a decrease in $\mathrm{H} 3$ and $\mathrm{H} 4$ acetylation was observed in the promoter of the gpt transgene in G12 cells [45]. TSA, with or without 5-aza-cytidine, is able to reactivate gpt gene silenced by nickel. Furthermore, exposure of nickel-transformed cells to TSA significantly reduced the transformation phenotype induced by nickel, suggesting that gene silencing mediated by histone modification may play a role in nickel-induced cell transformation [58]. It is worth noting that a recent study has shown that Ni can bind histone $\mathrm{H} 4$ tails and form a structure similar to lysine acetylation, which might prevent the lysine residues from further acetylation [59].

Methylation of $\mathrm{H} 3 \mathrm{~K} 9$ is normally associated with gene silencing. Cells exposed to nickel exhibited a significant increase in global levels of dimethylated H3K9 (H3K9me2) [60]. Chromatin immunoprecipitation confirmed the increase of $\mathrm{H} 3 \mathrm{~K} 9 \mathrm{me} 2$ in several gene-specific promoters, which likely contributes to the reduced expression of those genes [60,61]. Further studies demonstrated that the increase of $\mathrm{H} 3 \mathrm{~K} 9 \mathrm{me} 2$ was due to nickel inhibiting the activity of jmjc-domain containing demethylases [60,62]. Jmjc domain, which is a key structural motif shared by the members of non-heme dioxygenase superfamily, contains a catalytic site that binds the iron. The affinity constant of Ni (II) binding to this iron-binding motif is about three times greater than that of iron. Therefore, nickel is more likely to replace the iron at the iron-binding site, which permanently inhibits the demethylase activity [62]. Spry2, a downstream target of the H3K9me2 demethylase JMJD1A, was silenced by chronic exposure to nickel, and this potentiated nickel-induced anchorage-independent growth [61].

The investigations described above allude to a possible mechanism of Ni-induced carcinogenesis (Figure 1B). Nickel is able to modulate histone acetylation and methylation by targeting the enzymes that add or remove the marks. The changed enzyme activity as well as the histone marks may lead to altered gene expression and eventually contribute to nickel-induced carcinogenesis.

\section{Beyond Chromatin: microRNAs and Ni Induced Gene Silencing}

MicroRNAs (miRNAs) are endogenous small noncoding RNAs (18-25 nt) that negatively regulate gene expression in a sequence-specific manner [63,64]. These single-stranded RNAs bind to complementary sequences in $3^{\prime}$ or $5^{\prime}$ untranslated regions as well as coding regions of target mRNAs and result in mRNA degradation or inhibition of protein synthesis. According to the latest update of miRBase (version 19) [65], there are more than 2,000 unique mature miRNAs in the human genome, which are actively involved in many cellular processes including cell proliferation, differentiation, apoptosis, and metabolism [64]. Many studies have shown that miRNA profiles differ between normal and tumor tissues, and dysregulated miRNAs may play a crucial role in cancer initiation and progression. Because one mature miRNA is able to target multiple mRNAs due to binding of partially 
complementary regions, dysregulated miRNAs in cancer cells may lead to tremendous changes in gene expression.

Recent studies have reported that miRNAs may play a role in Ni-induced cell transformation and tumorigenesis. Expression of miR-222 was significantly up-regulated in rat rhabdomyosarcomas induced by an intramuscular injection of nickel subsulfide as well as in nickel-transformed 16HBE cells [66]. miR-222, which is able to target several important tumor suppressor genes including p27, p57 and PTEN, has been found to be increased in many human cancers. Both p27 and p57 are important negative regulators of the cell cycle. Thus, deregulated miR-222 and reduced expression of p27 and p57 may contribute to accelerated cell growth observed in Ni-induced tumors as well as transformed cells [66].

Similar to protein-coding genes, miRNA expression can be regulated by DNA methylation in their promoter regions. miR-152, a tumor suppressor microRNA targeting DNMT1, was significantly down-regulated in nickel sulfide-transformed 16HBE cells [67]. As a result, DNMT1 levels increased, which lead to elevated DNA methylation levels and enriched MeCP2 at the promoter of miR-152. Moreover, while ectopic expression of miR-152 in nickel sulfide-transformed cells inhibited cell proliferation, expressing anti-miR-152 in normal 16HBE cells resulted in increased cell proliferation and colony formation [67]. These results clearly demonstrate that down-regulation of miR-152 contributes to nickel sulfide-induced cell transformation.

\section{Conclusions}

In the past two decades, research aiming to characterize the carcinogenicity of $\mathrm{Ni}$ compounds have uncovered the epigenetic alteration induced by nickel exposure. Multiple lines of evidence have indicated the ability of nickel to perturb the epigenome. First, nickel has been shown to initiate chromatin condensation and to silence transgenes located near heterochromatin. In addition, nickel exposure has been associated with DNA hypermethylation and transcriptional repression of tumor suppressor genes both in vitro and in vivo. Moreover, nickel is able to modulate various histone modifications by targeting the enzymes that add or remove the specific marks. Lastly, nickel may interfere with microRNA network to degrade mRNA or block protein synthesis. These changes in DNA methylation, histone modification and miRNA network, along with condensed chromatin structure, create an aberrant epigenetic landscape and contribute to tumor initiation and progression.

\section{Acknowledgements}

This work was supported by grant ES00260 from the National Institute of Environmental Health Sciences and grant 4/00/00/252 from King Abdulaziz University (KAU).

\section{Conflicts of Interest}

The authors declare no conflict of interest. 


\section{References}

1. Polednak, A.P. Mortality among welders, including a group exposed to nickel oxides. Arch. Environ. Health (US) 1981, 36, 235-242.

2. Grimsrud, T.K.; Berge, S.R.; Resmann, F.; Norseth, T.; Andersen, A. Assessment of historical exposures in a nickel refinery in Norway. Scand. J. Work Environ. Health 2000, 26, 338-345.

3. Doll, R.; Morgan, L.G.; Speizer, F.E. Cancers of the lung and nasal sinuses in nickel workers. Br. J. Cancer 1970, 24, 623-632.

4. Roberts, R.S.; Julian, J.A.; Muir, D.C.; Shannon, H.S. Cancer mortality associated with the high-temperature oxidation of nickel subsulfide. IARC Sci. Publ. 1984, 53, $23-35$.

5. Roberts, R.S.; Julian, J.A.; Muir, D.C.; Shannon, H.S. A study of mortality in workers engaged in the mining, smelting, and refining of nickel. II. Mortality from cancer of the respiratory tract and kidney. Toxicol. Ind. Health (USA) 1989, 5, 975-993.

6. Grimsrud, T.K.; Berge, S.R.; Haldorsen, T.; Andersen, A. Exposure to different forms of nickel and risk of lung cancer. Am. J. Epidemiol. 2002, 156, 1123-1132.

7. Sunderman, F.W. Carcinogenicity of nickel compounds in animals. IARC Sci. Publ. 1984, 53, $127-142$.

8. Lumb, G.; Sunderman, F.W. The mechanism of malignant tumor induction by nickel subsulfide. Ann. Clin. Lab. Sci. 1988, 18, 353-366.

9. Kasprzak, K.S.; Sunderman, F.W.; Salnikow, K. Nickel carcinogenesis. Mutat. Res. 2003, 533, 67-97.

10. Patierno, S.R.; Dirscherl, L.A.; Xu, J. Transformation of rat tracheal epithelial cells to immortal growth variants by particulate and soluble nickel compounds. Mutat. Res. 1993, 300, 179-193.

11. Landolph, J.R. Molecular mechanisms of transformation of C3H/10T1/2 C1 8 mouse embryo cells and diploid human fibroblasts by carcinogenic metal compounds. Environ. Health Perspect. 1994, $102,119-125$.

12. Lu, H.; Shi, X.; Costa, M.; Huang, C. Carcinogenic effect of nickel compounds. Mol. Cell. Biochem. 2005, 279, 45-67.

13. Miura, T.; Patierno, S.R.; Sakuramoto, T.; Landolph, J.R. Morphological and neoplastic transformation of $\mathrm{C} 3 \mathrm{H} / 10 \mathrm{~T} 1 / 2 \mathrm{Cl} 8$ mouse embryo cells by insoluble carcinogenic nickel compounds. Environ. Mol. Mutagen. 1989, 14, 65-78.

14. IARC. Chromium, Nickel and Welding. IARC Monographs on the Evaluation of Carcinogenic Risks to Humans; World Health Organization: Lyon, France, 1990; Volume 49.

15. DOLL, R.; Andersen, A.; Cooper, W.; Cosmatos, I.; Cragle, D.; Easton, D.; Enterline, P.; Goldberg, M.; Metcalfe, L.; Norseth, T. Report of the international committee on nickel carcinogenesis in man. Scand. J. Work Environ. Health 1990, 16, 1-82.

16. Costa, M.; Davidson, T.L.; Chen, H.; Ke, Q.; Zhang, P.; Yan, Y.; Huang, C.; Kluz, T. Nickel carcinogenesis: Epigenetics and hypoxia signaling. Mutat. Res. 2005, 592, 79-88.

17. Salnikow, K.; Zhitkovich, A. Genetic and epigenetic mechanisms in metal carcinogenesis and cocarcinogenesis: Nickel, arsenic, and chromium. Chem. Res. Toxicol. 2008, 21, 28-44.

18. Chervona, Y.; Arita, A.; Costa, M. Carcinogenic metals and the epigenome: Understanding the effect of nickel, arsenic, and chromium. Metallomics 2012, 4, 619-627. 
19. Conway, K.; Costa, M. Nonrandom chromosomal alterations in nickel-transformed Chinese hamster embryo cells. Cancer Res. 1989, 49, 6032-6038.

20. Conway, K.; Costa, M. The involvement of heterochromatic damage in nickel-induced transformation. Biol. Trace Elem. Res. 1989, 21, 437-444.

21. Clemens, F.; Verma, R.; Ramnath, J.; Landolph, J.R. Amplification of the Ect2 proto-oncogene and over-expression of Ect 2 mRNA and protein in nickel compound and methylcholanthrenetransformed 10T1/2 mouse fibroblast cell lines. Toxicol. Appl. Pharmacol. 2005, 206, 138-149.

22. Salnikow, K.; Blagosklonny, M.V.; Ryan, H.; Johnson, R.; Costa, M. Carcinogenic nickel induces genes involved with hypoxic stress. Cancer Res. 2000, 60, 38-41.

23. Davidson, T.; Salnikow, K.; Costa, M. Hypoxia inducible factor-1 alpha-independent suppression of aryl hydrocarbon receptor-regulated genes by nickel. Mol. Pharmacol. 2003, 64, 1485-1493.

24. Salnikow, K.; Davidson, T.; Zhang, Q.; Chen, L.C.; Su, W.; Costa, M. The involvement of hypoxia-inducible transcription factor-1-dependent pathway in nickel carcinogenesis. Cancer Res. 2003, 63, 3524-3530.

25. Baylin, S.B.; Jones, P.A. A decade of exploring the cancer epigenomebiological and translational implications. Nat. Rev. Cancer 2011, 11, 726-734.

26. Dawson, M.A.; Kouzarides, T. Cancer epigenetics: From mechanism to therapy. Cell 2012, 150, $12-27$.

27. Arita, A.; Costa, M. Epigenetics in metal carcinogenesis: Nickel, arsenic, chromium and cadmium. Metallomics 2009, 1, 222-228.

28. You, J.S.; Jones, P.A. Cancer genetics and epigenetics: Two sides of the same coin? Cancer Cell 2012, 22, 9-20.

29. Latham, J.A.; Dent, S.Y.R. Cross-regulation of histone modifications. Nat. Struct. Mol. Biol. 2007, 14, 1017-1024.

30. Cohen, I.; Poręba, E.; Kamieniarz, K.; Schneider, R. Histone modifiers in cancer: Friends or foes? Genes Cancer 2011, 2, 631-647.

31. Greer, E.L.; Shi, Y. Histone methylation: A dynamic mark in health, disease and inheritance. Nat. Rev. Genet. 2012, 13, 343-357.

32. Ball, M.P.; Li, J.B.; Gao, Y.; Lee, J.-H.; LeProust, E.M.; Park, I.-H.; Xie, B.; Daley, G.Q.; Church, G.M. Targeted and genome-scale strategies reveal gene-body methylation signatures in human cells. Nat. Biotechnol. 2009, 27, 361-368.

33. Jones, P.A. Functions of DNA methylation: Islands, start sites, gene bodies and beyond. Nat. Rev. Genet. 2012, 13, 484-492.

34. Li, X.-Q.; Guo, Y.-Y.; De, W. DNA methylation and microRNAs in cancer. World J. Gastroenterol. 2012, 18, 882-888.

35. Wu, S.C.; Zhang, Y. Active DNA demethylation: Many roads lead to Rome. Nat. Rev. Mol. Cell Biol. 2010, 11, 607-620.

36. Klein, C.B.; Conway, K.; Wang, X.W.; Bhamra, R.K.; Lin, X.H.; Cohen, M.D.; Annab, L.; Barrett, J.C.; Costa, M. Senescence of nickel-transformed cells by an X chromosome: Possible epigenetic control. Science 1991, 251, 796-799.

37. Klein, C.B.; Rossman, T.G. Transgenic Chinese hamster V79 cell lines which exhibit variable levels of gpt mutagenesis. Environ. Mol. Mutagen. 1990, 16, 1-12. 
38. Kargacin, B.; Klein, C.B.; Costa, M. Mutagenic responses of nickel oxides and nickel sulfides in Chinese hamster V79 cell lines at the xanthine-guanine phosphoribosyl transferase locus. Mutat. Res. 1993, 300, 63-72.

39. Lee, Y.W.; Pons, C.; Tummolo, D.M.; Klein, C.B.; Rossman, T.G.; Christie, N.T. Mutagenicity of soluble and insoluble nickel compounds at the gpt locus in G12 Chinese hamster cells. Environ. Mol. Mutagen. 1993, 21, 365-371.

40. Verma, R.; Ramnath, J.; Clemens, F.; Kaspin, L.C.; Landolph, J.R. Molecular biology of nickel carcinogenesis: Identification of differentially expressed genes in morphologically transformed C3H10T1/2 $\mathrm{Cl} 8$ mouse embryo fibroblast cell lines induced by specific insoluble nickel compounds. Mol. Cell. Biochem. 2004, 255, 203-216.

41. Landolph, J.R.; Verma, A.; Ramnath, J.; Clemens, F. Molecular biology of deregulated gene expression in transformed $\mathrm{C} 3 \mathrm{H} / 10 \mathrm{~T} 1 / 2$ mouse embryo cell lines induced by specific insoluble carcinogenic nickel compounds. Environ. Health Perspect. 2002, 110, 845-850.

42. DeSilva, A.; Verma, R.; Landolph, J.R. Silencing of Expression the Beta Centaurin-2 and the FAD Synthetae Genes in Nickel Transformed C3H/10T1/2 Cell Lines. In Meal Ions in Biology and Medicine; Collery, P., Maymaud, I., Theophanides, T., Khassanova, I., Collery, T., Eds.; John Libbey Eurotext: Paris, France, 2008; Volume 10, pp. 65-67.

43. Lee, Y.W.; Klein, C.B.; Kargacin, B.; Salnikow, K.; Kitahara, J.; Dowjat, K.; Zhitkovich, A.; Christie, N.T.; Costa, M. Carcinogenic nickel silences gene expression by chromatin condensation and DNA methylation: A new model for epigenetic carcinogens. Mol. Cell. Biol. 1995, 15, 2547-2557.

44. Ellen, T.P.; Kluz, T.; Harder, M.E.; Xiong, J.; Costa, M. Heterochromatinization as a potential mechanism of nickel-induced carcinogenesis. Biochemistry 2009, 48, 4626-4632.

45. Yan, Y.; Kluz, T.; Zhang, P.; Chen, H.-B.; Costa, M. Analysis of specific lysine histone H3 and $\mathrm{H} 4$ acetylation and methylation status in clones of cells with a gene silenced by nickel exposure. Toxicol. Appl. Pharmacol. 2003, 190, 272-277.

46. Wu, C.-H.; Tang, S.-C.; Wang, P.-H.; Lee, H.; Ko, J.-L. Nickel-induced epithelial-mesenchymal transition by reactive oxygen species generation and E-cadherin promoter hypermethylation. J. Biol. Chem. 2012, 287, 25292-25302.

47. Ji, W.; Yang, L.; Yu, L.; Yuan, J.; Hu, D.; Zhang, W.; Yang, J.; Pang, Y.; Li, W.; Lu, J.; et al. Epigenetic silencing of $\mathrm{O}^{6}$-methylguanine DNA methyltransferase gene in NiS-transformed cells. Carcinogenesis 2008, 29, 1267-1275.

48. Yasaei, H.; Gilham, E.; Pickles, J.C.; Roberts, T.P.; O’Donovan, M.; Newbold, R.F. Carcinogen-specific mutational and epigenetic alterations in INK4A, INK4B and p53 tumor-suppressor genes drive induced senescence bypass in normal diploid mammalian cells. Oncogene 2013, 32, 171-179.

49. Govindarajan, B.; Klafter, R.; Miller, M.S.; Mansur, C.; Mizesko, M.; Bai, X.; LaMontagne, K.; Arbiser, J.L. Reactive oxygen-induced carcinogenesis causes hypermethylation of p16(Ink4a) and activation of MAP kinase. Mol. Med. 2002, 8, 1-8.

50. Zhang, J.; Zhang, J.; Li, M.; Wu, Y.; Fan, Y.; Zhou, Y.; Tan, L.; Shao, Z.; Shi, H. Methylation of RAR- $\beta 2$, RASSF1A, and CDKN2A genes induced by nickel subsulfide and nickel-carcinogenesis in rats. Biomed. Environ. Sci. 2011, 24, 163-171.

51. Broday, L.; Peng, W.; Kuo, M.H.; Salnikow, K.; Zoroddu, M.; Costa, M. Nickel compounds are novel inhibitors of histone H4 acetylation. Cancer Res. 2000, 60, 238-241. 
52. Golebiowski, F.; Kasprzak, K.S. Inhibition of core histones acetylation by carcinogenic nickel(II). Mol. Cell. Biochem. 2005, 279, 133-139.

53. Ke, Q.; Davidson, T.; Chen, H.; Kluz, T.; Costa, M. Alterations of histone modifications and transgene silencing by nickel chloride. Carcinogenesis 2006, 27, 1481-1488.

54. Kang, J.; Zhang, Y.; Chen, J.; Chen, H.; Lin, C.; Wang, Q.; Ou, Y. Nickel-induced histone hypoacetylation: The role of reactive oxygen species. Toxicol. Sci. 2003, 74, 279-286.

55. Chen, C.-Y.; Wang, Y.-F.; Huang, W.-R.; Huang, Y.-T. Nickel induces oxidative stress and genotoxicity in human lymphocytes. Toxicol. Appl. Pharmacol. 2003, 189, 153-159.

56. Salnikow, K.; Gao, M.; Voitkun, V.; Huang, X.; Costa, M. Altered oxidative stress responses in nickel-resistant mammalian cells. Cancer Res. 1994, 54, 6407-6412.

57. Sutherland, J.E.; Peng, W.; Zhang, Q.; Costa, M. The histone deacetylase inhibitor trichostatin A reduces nickel-induced gene silencing in yeast and mammalian cells. Mutat. Res. 2001, 479, 225-233.

58. Zhang, Q.; Salnikow, K.; Kluz, T.; Chen, L.C.; Su, W.C.; Costa, M. Inhibition and reversal of nickel-induced transformation by the histone deacetylase inhibitor trichostatin A. Toxicol. Appl. Pharmacol. 2003, 192, 201-211.

59. Zoroddu, M.A.; Peana, M.; Medici, S.; Casella, L.; Monzani, E.; Costa, M. Nickel binding to histone H4. Dalton Trans. 2010, 39, 787-793.

60. Chen, H.; Ke, Q.; Kluz, T.; Yan, Y.; Costa, M. Nickel ions increase histone H3 lysine 9 dimethylation and induce transgene silencing. Mol. Cell. Biol. 2006, 26, 3728-3737.

61. Chen, H.; Kluz, T.; Zhang, R.; Costa, M. Hypoxia and nickel inhibit histone demethylase JMJD1A and repress Spry2 expression in human bronchial epithelial BEAS-2B cells. Carcinogenesis 2010, 31, 2136-2144.

62. Chen, H.; Giri, N.C.; Zhang, R.; Yamane, K.; Zhang, Y.; Maroney, M.; Costa, M. Nickel ions inhibit histone demethylase JMJD1A and DNA repair enzyme ABH2 by replacing the ferrous iron in the catalytic centers. J. Biol. Chem. 2010, 285, 7374-7383.

63. He, L.; Hannon, G.J. MicroRNAs: Small RNAs with a big role in gene regulation. Nat. Rev. Genet. 2004, 5, 522-531.

64. Bartel, D.P. MicroRNAs: Target recognition and regulatory functions. Cell 2009, 136, 215-233.

65. Kozomara, A.; Griffiths-Jones, S. miRBase: Integrating microRNA annotation and deep-sequencing data. Nucleic Acids Res. 2011, 39, D152-D157.

66. Zhang, J.; Zhou, Y.; Ma, L.; Huang, S.; Wang, R.; Gao, R.; Wu, Y.; Shi, H.; Zhang, J. The alteration of MiR-222 and its target genes in nickel-induced tumor. Biol. Trace Elem. Res. 2013, 152, 267-274.

67. Ji, W.; Yang, L.; Yuan, J.; Yang, L.; Zhang, M.; Qi, D.; Duan, X.; Xuan, A.; Zhang, W.; Lu, J.; et al. MicroRNA-152 targets DNA methyltransferase 1 in NiS-transformed cells via a feedback mechanism. Carcinogenesis 2013, 34, 446-453.

(C) 2013 by the authors; licensee MDPI, Basel, Switzerland. This article is an open access article distributed under the terms and conditions of the Creative Commons Attribution license (http://creativecommons.org/licenses/by/3.0/). 